\title{
Medicina Intensiva na Graduação Médica: Perspectiva do Estudante*
}

\section{Intensive Care Medicine on Medical Undergraduation: Student's Perspective}

\author{
Alessandro de Moura Almeida', Ligia Carvalho Albuquerque², Almir Galvão Vieira Bitencourt', \\ Carlos Eduardo Cerqueira Rolim², Tiana Mascarenhas Godinho', Maurício Valverde Liberato', \\ Fernando Cezar Cabral Oliveira Filho², Ana Bárbara Galvão de Azevedo', Ana Paula Soares da Silva Neves', \\ Marcelo de Jesus Martins ${ }^{1}$, João Paulo Maciel Silva ${ }^{1}$, Paulo André Jesuíno ${ }^{3}$, Sydney Agareno de Souza Filho ${ }^{3}$
}

\section{RESUMO}

JUSTIFICATIVA E OBJETIVOS: Acredita-se que existe uma deficiência no ensino de Medicina Intensiva (MI) na graduação da maioria das escolas médicas, levando a um prejuízo na formação acadêmica de futuros médicos. O objetivo deste estudo foi analisar o ensino e o grau de interesse em MI por estudantes de Medicina de Salvador-BA.

MÉTODO: Estudo transversal realizado em 2005 entre estudantes do $6^{\circ}$ ao $12^{\circ}$ semestres de duas escolas médicas baianas. Utilizou-se um questionário autoaplicável composto de questões objetivas sobre interesse, habilidades e conhecimentos dos estudantes sobre Ml, bem como a sua opinião sobre o ensino dessa especialidade em sua faculdade.

RESULTADOS: Foram entrevistados 570 estudantes. A maioria $(57,5 \%)$ nunca realizou estágio em unidades de terapia intensiva (UTI). Contudo, a utilidade deste para o futuro profissional de um médico foi

1. Médico Membro Fundador da Liga Acadêmica de Medicina da Bahia (LAMIB)

2. Acadêmico de Medicina. Membro Fundador da Liga Acadêmica de Medicina da Bahia (LAMIB)

3. Médico Intensivista. Membro Consultor da Liga Acadêmica de Medicina da Bahia (LAMIB).

*Recebido da Liga Acadêmica de Medicina da Bahia (LAMIB), Salvador, $\mathrm{BA}$

Apresentado em 11 de setembro de 2007

Aceito para publicação em 15 de outubro de 2007

Endereço para correspondência:

Dr. Alessandro de Moura Almeida

Rua Nossa Senhora de Brotas, 107. Fauna - 703 Brotas

40285-170 Salvador, BA

Fones: (71) 3357-5459 / 9956-1786

E-mail: alessandrodemoura@yahoo.com.br

(C)Associação de Medicina Intensiva Brasileira, 2007 classificada como alta (média de 4,14 $\pm 1,05$, numa escala de 1 a 5) pelos entrevistados. O interesse em $\mathrm{MI}$ foi considerado alto ou muito alto por $53,7 \%$ da amostra. Quase todos os alunos (97\%) acreditam que tópicos de $\mathrm{Ml}$ devam ser mais explorados em seus currículos. Apenas 42,1\% sentiam-se seguros em avaliar um paciente gravemente enfermo, sendo essa segurança maior entre aqueles que já realizaram estágios em UTI $(p<0,001)$. Os tópicos de MI de maior interesse foram choque, reanimação cardiopulmonar e sepse.

CONCLUSÕES: O presente estudo revelou um alto interesse em Medicina Intensiva entre estudantes de Medicina da Bahia. Contudo, a maioria nunca realizou estágio em UTI, o que demonstrou ser importante fator na segurança do médico em formação frente ao paciente gravemente enfermo.

Unitermos: Educação, Estudantes de Medicina, UTI

\section{SUMMARY}

BACKGROUND AND OBJECTIVES: There are deficiencies on Intensive Medicine (IM) teaching in most of medical undergraduate schools. Those deficiencies may imply damages on their clinical competence. The objective of this study was to analyze current status of IM teaching and the medical undergraduate student interest in this speciality.

METHODS: A cross-sectional study was performed in 2005. We applied a self-reported questionnaire to enrolled students between the sixth and the last semesters of two medical schools from Salvador-Bahia. The questionnaire contained questions about students' interest and knowledge on IM, and opinion on IM teaching in their schools.

RESULTS: We studied 570 students. Most of them $(57.5 \%)$ had never realized a clerkship in intensive care unit (ICU) despite classifying its usefulness as high 
(mean of $4.14 \pm 1.05$, in a scale from 1 to 5 ). IM interest was high or very high in $53.7 \%$ of sample. Almost all students (97\%) thought that IM topics should be more explored at their curriculum. Only $42.1 \%$ reported to be able to assess a critical care patient and this assurance was higher among students with previous clerkship in ICU ( $p<0.001)$. Shock, cardiopulmonary resuscitation and sepsis were the most interesting topics in ICU for students' opinion.

CONCLUSIONS: This study revealed a high interest in IM among medical undergraduate students. However, most had never practice a clerkship in ICU, demonstrating to be an important factor on undergraduate student performance faced to a critical care patient.

Key Words: Education, ICU, Medical Students.

\section{INTRODUÇÃO}

A Medicina Intensiva (MI) desenvolveu-se do avanço de muitas disciplinas clínicas. Sua história vem do reconhecimento de que certos pacientes graves poderiam ter melhor atendimento, caso fossem reunidos em áreas específicas de um hospital, onde seriam assistidos por uma equipe multidisciplinar especializada. A engenharia dos hospitais para a construção dessas unidades semifechadas denominadas unidades de terapia intensiva (UTI), deu-se inicio a partir dos anos $1960^{1}$. Desta forma, surgiu uma especialidade que abrange atitudes e habilidades no reconhecimento e manuseio de pacientes gravemente enfermos, agrupando conhecimentos de diversas áreas da Medicina como Clínica Médica, Cirurgia, Anestesiologia e Pediatria ${ }^{1,2}$.

A partir de então, houve um rápido avanço no conhecimento em $\mathrm{Ml}^{3}$, que hoje é uma especialidade médica reconhecida pelo Conselho Federal de Medicina. Contudo, existe ainda uma deficiência na transferência dos conhecimentos dessa especialidade para alunos de graduação da maioria das escolas médicas ${ }^{2}$. No Brasil, a Ml é incluída no currículo de apenas algumas escolas médicas ${ }^{4,5}$.

Existem muitas razões para a necessidade de assegurar que médicos tenham, no mínimo, alguma familiaridade com bases da Medicina intensiva, que incluem o reconhecimento precoce de uma condição grave e o estabelecimento de intervenções de suporte antes da transferência para a UTI, além da habilidade em referenciar adequadamente um pacientes aos cuidados intensivos ${ }^{6}$. Nas escolas médicas da Bahia, historicamente, os acadêmicos de Medicina buscam seu aprendizado nos temas relacionados à $\mathrm{Ml}$ apenas através de eventos como congressos e simpósios, além de estágios extracurriculares.

O objetivo deste estudo foi analisar o status do ensino e o grau de interesse em MI por estudantes das faculdades de Medicina de Salvador-BA.

\section{MÉTODO}

Após aprovação pelo Comitê de Ética em Pesquisa da Maternidade Climério de Oliveira (CEP-MCO/ HUPESUFBA), foi realizado um estudo de corte transversal durante o ano de 2005. Todos os participantes do estudo assinaram consentimento informado sobre sua participação.

Foi utilizado um questionário auto-aplicável entregue aos estudantes regularmente matriculados do $6^{\circ}$ ao $12^{\circ}$ segundo semestres da Faculdade de Medicina da Universidade Federal da Bahia (pública) e da Escola Bahiana de Medicina e Saúde Pública (privada). Nenhuma das escolas estudadas possui a disciplina de Medicina Intensiva em sua grade curricular. O processo de entrevista foi supervisionado por membros da Liga Acadêmica de Medicina Intensiva da Bahia (LAMIB). Os supervisores não puderam tecer nenhum tipo de comentário que pudesse de alguma forma influenciar em qualquer resposta do participante da pesquisa.

O questionário era composto de questões objetivas sobre dados de identificação (idade, sexo, semestre letivo e faculdade de origem), interesses, habilidades e conhecimentos dos estudantes de Medicina sobre MI, bem como a sua opinião sobre o ensino em sua faculdade. Os entrevistados foram questionados sobre a sua habilidade em realizar procedimentos (acesso venoso periférico, colocação de cateter de artéria pulmonar, intubação traqueal, toracocentese, traqueostomia, passagem de sonda vesical, acesso venoso central, reanimação cardiopulmonar, passagem de sonda nasogástrica e drenagem torácica) rotineiros em UTI. Além disso, solicitou-se que o entrevistado classificasse, numa escala de 1 a 5 , seu grau de interesse, além das abordagens teórica e prática durante sua graduação, nos seguintes tópicos: reanimação cardiopulmonar, choque, monitorização hemodinâmica, atendimento inicial ao politraumatizado, síndrome da resposta inflamatória sistêmica (SIRS)/sepse, insuficiência respiratória e ventilação mecânica, distúrbios hidroeletrolíticos e metabólicos, sedação e analgesia, infecção em pacientes críticos, nutrição em pacientes críticos, insuficiência 
renal aguda e diálise, morte encefálica e transplante de órgãos, ética e humanização no paciente terminal, atendimento multidisciplinar em UTI. Para efeito de análise, o estudante foi incluído no estudo somente se respondeu, no mínimo, metade das questões. Foram anulados as questões ou os questionários que estivessem rasurados ou que não permitissem a exata identificação da resposta dada.

Para tabulação e análise dos dados, utilizou-se o programa SPSS (Statistical Package for Social Science), versão 9.0 (SPSS Inc. Chicago, Illnois). Foi realizada a análise descritiva das variáveis, fazendo as correlações adequadas, teste $t$ de Student para análise de variáveis contínuas nos grupos estudados e o teste do Qui-quadrado para análise de proporções. O erro do tipo I foi considerado como $5 \%$ para todas as análises.

\section{RESULTADOS}

Participaram do estudo 570 graduandos de Medicina. As características demográficas estão apresentadas na tabela 1. A maioria dos alunos $(57,5 \%, \mathrm{n}=328)$ nunca havia freqüentado alguma UTI, sendo que eles classificaram como alta (média de 4,14 $\pm 1,05$, numa escala de 1 a 5) a utilidade de um estágio nesta área para seu futuro profissional. Os alunos que já haviam freqüentado algum estágio em UTI classificaram com escores mais elevados a sua utilidade para seu futuro profissional $(4,29 \pm 0,96$ versus $4,03 \pm 1,10 ; p=0,004)$. Não foi demonstrada diferença entre a classificação dos alunos que realizaram estágios curriculares $(4,28$ $\pm 0,73$ ), se comparados com aqueles que realizaram estágios extracurriculares $(4,29 \pm 0,99)(p=0,947)$.

\begin{tabular}{lccc}
\multicolumn{4}{c}{ Tabela 1 - Características Demográficas dos Estudantes } \\
\hline Características & $\begin{array}{c}\text { Escola Pública } \\
(\mathrm{n}=216)\end{array}$ & $\begin{array}{c}\text { Escola Privada } \\
(\mathrm{n}=354)\end{array}$ & $\begin{array}{c}\text { Total } \\
(\mathrm{n}=570)\end{array}$ \\
\hline Sexo $-\mathrm{n}(\%)$ & $129(59,8)$ & $132(37,3)$ & $261(45,8)$ \\
$\quad$ Masculino & $80(37,0)$ & $179(50,6)$ & $259(45,4)$ \\
Feminino & $7(3,2)$ & $43(12,1)$ & $50(8,8)$ \\
$\quad$ Não anotado & & & \\
Idade (anos) & $22,8 \pm 1,7$ & $22,9 \pm 1,8$ & $22,9 \pm 1,8$ \\
Média \pm DP & 23 & 23 & 23 \\
Mediana & $19 \longmapsto 28$ & $19 \longmapsto 33$ & $19 \longmapsto 33$ \\
Limites & & & \\
Semestre - $\mathrm{n}(\%)$ & $41(19,0)$ & $61(17,2)$ & $102(17,9)$ \\
Sexto & $56(25,9)$ & $80(22,6)$ & $136(23,9)$ \\
Sétimo & $65(30,1)$ & $53(15,0)$ & $118(20,7)$ \\
Oitavo & $54(25,0)$ & $158(44,6)$ & $212(37,1)$ \\
Internato & $0(0)$ & $2(0,6)$ & $2(0,4)$ \\
Não anotado & &
\end{tabular}

DP = Desvio-padrão
Entre aqueles que não tinham realizado estágio em terapia intensiva, o principal motivo foi a falta de oportunidade em fazê-lo $(75,3 \%)$, seguido de falta de tempo disponível (12,3\%). Apenas 6,3\% desses estudantes referiram que não realizaram estágio por falta de interesse. Já aqueles que já haviam freqüentado alguma UTI $(86,1 \%$ destes em atividades extracurriculares apenas) foram movidos principalmente por interesse da Medicina Intensiva como futura especialidade $(40,7 \%)$. A melhoria do currículo $(23,6 \%)$ e a oportunidade em realizar procedimentos $(22,5 \%)$ foram motivos também freqüentes entre os entrevistados.

$\mathrm{O}$ interesse na área de Medicina Intensiva foi considerado alto ou muito alto por $53,7 \%(n=306)$ da amostra. Apenas 11 (1,9\%) estudantes referiram não ter interesse em Medicina Intensiva. Este interesse foi maior entre aqueles que já haviam freqüentado estágio em UTI, conforme demonstrado na tabela 2.

Tabela 2 - Interesse em Medicina Intensiva entre os Estudantes de Medicina com e sem Estágio em Unidades de Terapia Intensiva $(n=552)$ *.

\begin{tabular}{|c|c|c|c|}
\hline \multirow{2}{*}{$\begin{array}{l}\text { Interesse na Área de } \\
\text { Medicina Intensiva }\end{array}$} & \multicolumn{2}{|c|}{ Realizou Estágio em UTI? } & \multirow{2}{*}{ Total - n (\%) } \\
\hline & Não - n (\%) & $\operatorname{Sim}(\%)$ & \\
\hline Nenhum & $6(1,9)$ & $1(0,4)$ & $7(1,3)$ \\
\hline Baixo & $38(11,8)$ & $18(7,9)$ & $56(10,1)$ \\
\hline Médio & $127(39,3)$ & $57(24,9)$ & $184(33,3)$ \\
\hline Alto & $109(33,7)$ & $88(38,4)$ & $197(35,7)$ \\
\hline Muito alto & $43(13,3)$ & $65(28,4)$ & $108(19,6)$ \\
\hline Total & $323(100)$ & $229(100)$ & $552(100)$ \\
\hline
\end{tabular}

${ }^{*} \mathrm{p}<0,001$

Quase todos os alunos (97\%, $n=553$ ) acreditam que tópicos de Medicina Intensiva devam ser mais explorados em seus currículos. Os principais problemas do ensino da especialidade em suas escolas foram, em suas opiniões, desinteresse da diretoria ou coordenação $(52,1 \%, n=297)$, inexistência de uma UTI $(22,6 \%$, $n=129)$, desinteresse dos professores $(10,9 \%, n=62)$ e falta de professores qualificados $(9,1 \%, n=52)$.

Embora a maioria dos alunos $(58,2 \%, n=332)$ já tenha participado da indicação de um paciente para UTI, uma parte deles $(34,8 \%)$ não se acha capaz de identificar um paciente com necessidade de cuidados intensivos. Apenas $42,1 \%(n=240)$ sentem-se seguro em avaliar um paciente internado numa UTI, sendo essa segurança maior entre os estudantes que já realizaram estágios nessa área $(56,4 \%$ versus $32,6 \% ; p<0,001)$. Observou-se ainda maior segurança tanto na identificação do paciente com necessidade de tratamento 
intensivo, quanto na avaliação deste paciente em UTI com o avançar do curso médico (Tabela 3).

A realização de procedimentos e o contato curricular dos estudantes com tópicos relacionados à Ml podem ser vistos nas tabelas 4 e 5 . O grau de interesse (classificado numa escala de 1 a 5 ) dos estudantes nos principais tópicos de $\mathrm{Ml}$ foi: choque $(4,69 \pm 0,66)$; RCP $(4,69 \pm 0,67) ;$ SIRS/Sepse $(4,58 \pm 0,75)$; atendimento inicial ao politraumatizado $(4,51 \pm 0,89)$; insuficiência respiratória e ventilação mecânica $(4,50 \pm 0,80)$; distúrbios hidroeletrolíticos e metabólicos $(4,47 \pm 0,84)$; insuficiência renal aguda e métodos dialíticos $(4,40 \pm$ $0,91)$; sedação e analgesia $(4,39 \pm 0,86)$; monitorização hemodinâmica $(4,38 \pm 0,90)$; infecção em pacientes críticos $(4,36 \pm 0,92)$; morte encefálica e transplante de órgãos $(4,31 \pm 1,00)$; ética e humanização em UTI $(4,27$ $\pm 1,03)$; atendimento multidisciplinar em UTI $(4,23 \pm$ $1,01)$; e nutrição no paciente crítico $(4,01 \pm 1,08)$.

Tabela 3 - Segurança na Identificação de Indicação de UTI (A) e na Avaliação (B) de Paciente Internado em UTI. Segundo Semestre do Curso e Escola Estudada.

\begin{tabular}{|c|c|c|c|c|c|c|}
\hline \multirow{2}{*}{ Semestre } & \multicolumn{2}{|c|}{ Escola Pública (\%) } & \multicolumn{2}{|c|}{ Escola Privada (\%) } & \multicolumn{2}{|c|}{ Total (\%) } \\
\hline & $A^{*}$ & $\mathrm{~B}^{*}$ & $A^{*}$ & $\mathrm{~B}^{\star}$ & $A^{*}$ & $\mathrm{~B}^{*}$ \\
\hline Sexto & 7,7 & 9,8 & 39,7 & 21,3 & 26,8 & 16,7 \\
\hline Sétimo & 22,2 & 20,4 & 33,8 & 30,0 & 28,9 & 26,1 \\
\hline Oitavo & 58,1 & 49,2 & 46,0 & 39,6 & 52,7 & 44,9 \\
\hline Internato & 83,3 & 67,9 & 75,0 & 63,2 & 77,0 & 64,4 \\
\hline Total & 44,8 & 39,0 & 55,4 & 44,7 & 51,4 & 42,5 \\
\hline
\end{tabular}

${ }^{*} \mathrm{p}<0,001$

Tabela 4 - Realização de Procedimentos Supervisionados em UTI por Estudantes de Medicina do Segundo Semestre

\begin{tabular}{|c|c|c|c|c|c|}
\hline Procedimentos & Sexto Semestre (\%) & Sétimo Semestre (\%) & Oitavo Semestre (\%) & Internato (\%) & Total (\%) \\
\hline Acesso venoso periférico* & 16,7 & 20,0 & 37,3 & 80,0 & 46,6 \\
\hline Acesso venoso central $^{*}$ & 2,0 & 2,2 & 12,7 & 54,2 & 24,1 \\
\hline Cateter de artéria pulmonar* & 1,0 & 2,2 & 5,9 & 13,2 & 7,0 \\
\hline Intubação traqueal* & 5,9 & 6,0 & 15,3 & 45,8 & 22,9 \\
\hline Traqueostomia* & 2,0 & 3,7 & 2,5 & 16,5 & 8,1 \\
\hline Toracocentese ${ }^{\star}$ & 2,0 & 3,7 & 3,4 & 22,6 & 10,5 \\
\hline Reanimação cardiorrespiratória* & 9,8 & 12,6 & 24,6 & 51,9 & 29,3 \\
\hline Drenagem torácica* & 2,9 & 3,7 & 7,6 & 30,7 & 14,6 \\
\hline Sonda vesical ${ }^{\star}$ & 11,8 & 14,8 & 20,3 & 67,0 & 35,0 \\
\hline Sonda nasogástrica* & 2,9 & 8,9 & 16,1 & 53,3 & 26,2 \\
\hline
\end{tabular}

${ }^{*} \mathrm{p}<0,001$

Tabela 5 - Contato Prévio dos Estudantes de Medicina com Tópicos Relacionados à Medicina Intensiva em Atividades Curriculares Teóricas $(T)$ e Práticas $(P)$.

\begin{tabular}{|c|c|c|c|c|c|c|c|c|c|c|}
\hline \multirow[t]{2}{*}{ Tópicos de Medicina Intensiva } & \multicolumn{2}{|c|}{$\begin{array}{c}\text { Sexto Semestre } \\
(\%)\end{array}$} & \multicolumn{2}{|c|}{$\begin{array}{c}\text { Sétimo Semestre } \\
(\%)\end{array}$} & \multicolumn{2}{|c|}{$\begin{array}{c}\text { Oitavo Semestre } \\
(\%)\end{array}$} & \multicolumn{2}{|c|}{ Internato (\%) } & \multicolumn{2}{|c|}{ Total (\%) } \\
\hline & $\mathrm{T}$ & $\mathrm{P}$ & $\mathrm{T}$ & $\mathrm{P}$ & $\mathrm{T}$ & $\mathrm{P}$ & $\mathrm{T}$ & $\mathrm{P}$ & $\mathrm{T}$ & $\mathrm{P}$ \\
\hline Reanimação cardiopulmonar* & 91,5 & 49,5 & 87,6 & 37,5 & 98,2 & 28,2 & 97,5 & 48,1 & 94,3 & 41,8 \\
\hline Choque* & 73,4 & 6,5 & 77,5 & 6,0 & 99,1 & 12,8 & 98,0 & 31,9 & 89,1 & 17,2 \\
\hline Monitorização hemodinâmica* & 26,4 & 7,7 & 30,8 & 4,3 & 68,8 & 14,7 & 66,2 & 22,2 & 51,8 & 13,9 \\
\hline Atendimento inicial ao politraumatizado* & 76,1 & 18,5 & 69,1 & 13,9 & 99,1 & 15,5 & 98,0 & 50,3 & 87,6 & 28,5 \\
\hline SIRS/Sepse* & 72,6 & 5,4 & 82,6 & 14,0 & 99,1 & 15,6 & 96,9 & 34,2 & 89,7 & 20,3 \\
\hline Insuficiência respiratória e ventilação mecânica* & 52,1 & 11,1 & 66,9 & 12,5 & 74,5 & 14,4 & 67,9 & 24,2 & 66,3 & 17,1 \\
\hline Distúrbios hidroeletrolíticos e metabólicos ${ }^{\star}$ & 66,3 & 2,2 & 73,3 & 10,1 & 97,3 & 21,8 & 94,9 & 32,8 & 85,4 & 19,7 \\
\hline Sedação e analgesia** & 83,0 & 17,8 & 66,7 & 10,9 & 47,3 & 14,5 & 60,1 & 23,2 & 63,2 & 17,6 \\
\hline Infecção em pacientes críticos* & 42,1 & 0 & 46,7 & 5,8 & 53,2 & 13,5 & 66,0 & 20,9 & 54,7 & 12,3 \\
\hline Nutrição em pacientes críticos* & 14,0 & 3,4 & 41,7 & 4,1 & 46,8 & 4,6 & 75,5 & 18,0 & 50,6 & 9,4 \\
\hline Insuficiência renal aguda e diálise\# & 64,8 & 6,8 & 79,2 & 14,0 & 70,9 & 13,8 & 75,8 & 18,2 & 73,5 & 14,2 \\
\hline Morte encefálica e transplante de órgãos* & 48,4 & 1,1 & 46,7 & 3,3 & 93,6 & 3,7 & 64,1 & 11,2 & 63,1 & 6,1 \\
\hline Ética e humanização em UTI ${ }^{\star \star}$ & 71,6 & 11,0 & 64,8 & 7,4 & 45,0 & 7,3 & 60,8 & 17,6 & 60,3 & 11,9 \\
\hline Atendimento multidisciplinar em UTI** & 13,8 & 1,1 & 15,4 & 1,7 & 23,4 & 2,8 & 25,4 & 9,1 & 20,7 & 4,7 \\
\hline
\end{tabular}

${ }^{*} \mathrm{p} \leq 0,01$

${ }^{* *} \mathrm{p}<0,05$

\#Não significativo 


\section{DISCUSSÃO}

O presente estudo mostrou elevado interesse dos alunos em Ml, apesar da maioria não ter freqüentado alguma UTI. Entre os que já tinham freqüentado, grande parte o fez em unidades fora de seu ambiente acadêmico. Estudos realizados nos estados do Pará e do Rio de Janeiro mostraram que a procura por estágio extracurricular em universidades brasileiras pelos alunos do curso de Medicina é grande, principalmente em emergência e $\mathrm{UTI}^{7,8}$. As principais razões da procura por estágios extracurriculares são a busca por atividades práticas e por experiência clínica em atividades usuais da prática médica ${ }^{4}$. Segundo as Diretrizes Curriculares para os cursos de graduação em Medicina, o graduado deve saber atuar nos diferentes níveis de atendimento à saúde, de forma integral e interdisciplinar, promover a saúde, prevenir e tratar a doença e reabilitar a incapacidade ${ }^{9}$. Deste modo, é fundamental que o ensino de MI seja incorporado ao currículo de graduação das escolas médicas brasileiras. A participação em estágio em UTI auxilia nas formações generalistas e humanistas do médico, que vivencia experiências sobre fisiopatologia, terapêutica, ética, equipe multidisciplinar, contato com a morte e comunicação com o próprio paciente e seus familiares ${ }^{4}$.

Um estudo realizado em escolas médicas de língua inglesa ao redor do mundo mostrou que apenas cinco escolas (4\%) das participantes do estudo (122 escolas) não possuem Medicina intensiva em seu currículo ${ }^{10}$. Este mesmo estudo demonstrou que, embora $84 \%$ das escolas considerassem o ensino da Ml essencial na graduação médica, em apenas $31 \%$ delas este ensino é obrigatório ${ }^{10}$. A abordagem da Medicina Intensiva é, em alguns países, realizada preferencialmente em alunos de pós-graduação ${ }^{4}$. Como conseqüência dessa deficiência, os estudantes normalmente se graduam revelando carência na maioria das habilidades básicas inerentes à Terapia Intensiva, tendo dificuldades em referenciar apropriadamente um paciente para UTI e de iniciar intervenções em situação de emergência, conforme já descrito. Embora os resultados deste estudo demonstrem um aumento esperado na segurança do estudante quanto à indicação de cuidados intensivos e avaliação de pacientes críticos com o avançar do curso, os valores relatados pelos estudantes do internato ainda não são ideais, retratando possivelmente deficiência similar à observada em séries anteriores. O grau de interesse e a procura dos estudantes por estágios extracurriculares podem ter justificado o au- mento observado. Buchman e col. demonstraram que recém-graduados em Medicina não são capazes de realizar intervenções de suporte básico e avançado de vida, havendo um intervalo entre o que os estudantes aprendem e o que eles devem saber para iniciar efetivamente intervenções de Medicina crítica e para referir pacientes apropriadamente para UTI6. Tal insegurança pode ser diminuída pela inserção dos acadêmicos em estágios supervisionados onde eles possam vivenciar situações de manuseio de pacientes críticos, conforme observado por Rogers e col. ${ }^{11}$. Estes autores avaliaram um grupo de estudantes antes e após um estágio opcional em UTI e verificaram que após o estágio houve melhora na performance dos estudantes tanto em avaliação escrita, como em exame clínico objetivado e estruturado (OSCE) e em simulações com manequins ${ }^{11}$. Estes resultados demonstraram a importância deste tipo de atividade no desenvolvimento de habilidades e conhecimentos pelos médicos em formação. Além dos conhecimentos próprios da $\mathrm{MI}$, a conjugação imediata da terapêutica aos resultados com base em alterações fisiológicas, encontradas em muitas situações vivenciadas em UTI, leva o aluno a exercitar seus conhecimentos de Medicina básica e as aplicações na prática clínica, criando uma oportunidade de educação ampla e percepção da importância do papel do médico na questão técnica, humana e bioética frente à sociedade ${ }^{12}$.

Neste estudo, os estudantes consideraram importante a realização de um estágio em UTI para sua formação e a falta de oportunidade foi o principal motivo apontado pelos estudantes que não haviam realizado este tipo de estágio. O Comitê de Educação Médica da Society of Critical Care Medicine recomenda que todo estudante de Medicina seja introduzido aos aspectos clínicos e éticos da Ml através de, pelo menos, um rodízio em um serviço de terapia intensiva ${ }^{13}$. A realização deste tipo de estágio durante a graduação poderá promover maior incentivo para a escolha da MI como futura especialidade pelos estudantes. Atualmente há um déficit de especialistas nessa área em relação ao crescente número de serviços que vem sendo criados em todo o mundo, inclusive no Brasil. Angus e col. estimaram que se não ocorrer um aumento significativo no número de intensivistas, haverá uma deficiência de especialistas em MI nos Estados Unidos em torno de $22 \%$ da demanda em 2020 e 35\% em 2030 ${ }^{14}$. Grande parte dos estudantes avaliados referiu que buscou estágios extracurriculares em Ml pelo interesse em seguir esta especialidade no futuro, o que reforça a hipótese 
de que um aprimoramento no ensino desta disciplina na graduação possa incentivar a formação de novos intensivistas.

Shen e col. destacaram como os principais tópicos de Ml que devem ser incluídos no ensino dessa disciplina durante a graduação: RCP; avaliação e manuseio de pacientes agudos graves; condutas em disfunção de múltiplos órgãos incluindo insuficiência respiratória, choque, SIRS e sepse; manuseio de pacientes inconscientes; cuidados pós-operatórios imediatos; habilidades para comunicação e ética nas questões relacionadas aos pacientes terminais ${ }^{10}$. Muitos desses temas não são exclusivos da Ml e trazem estreita relação com outras áreas afins como Anestesiologia e emergência. Além disso, o conceito atual da $\mathrm{Ml}$ abrange o cuidado do paciente crítico desde o atendimento pré-hospitalar, o que vem trazendo importante diminuição de mortalidade deste paciente tanto na UTI quanto após sua alta.

Os resultados do presente estudo mostraram que os estudantes avaliados demonstraram grande interesse por todos os temas relacionados à Ml, no entanto tem contato restrito com alguns desses temas na faculdade, principalmente na sua abordagem prática, reforçando a hipótese de que o aumento observado na confiança adquirida pelo estudante na avaliação do paciente crítico deve-se, sobretudo, a experiências vividas em estágios extracurriculares.

A realização de procedimentos invasivos é outro ponto importante relacionado ao ensino da MI. Nossos dados sugerem que a crescente experiência observada na realização de procedimentos invasivos durante o curso se dá principalmente em atividades extracurriculares. Uma vez que é realizado fora do ambiente acadêmico das escolas médicas, estas atividades muitas vezes podem não ser adequadamente supervisionadas, acarretando na aprendizagem de técnicas e conceitos errados, além de condutas profissionais antiéticas que serão incorporadas e repetidas no exercício profissional futuro ${ }^{7}$. Pode-se perceber ainda que mesmo entre os alunos do internato, grande parcela não teve oportunidade de realizar, sob supervisão, alguns procedimentos considerados básicos na Ml como RCP, intubação traqueal e acesso venoso central. Para tentar suprir mais esta deficiência, a Associação de Medicina Intensiva Brasileira (AMIB), através do seu Comitê Especial das Ligas Acadêmicas de Medicina Intensiva (LIGAMI) vêm incentivando a criação de ligas de Medicina Intensiva por acadêmicos de Medicina, com a finalidade de maior inserção desta especialidade nas escolas médica brasileiras. Além disso, o citado comitê criou o "Curso de Introdução à Medicina Intensiva", onde são abordados aspectos básicos teórico-práticos comuns à $\mathrm{Ml}$ como intubação traqueal, RCP, acessos venosos profundos, acessos arteriais, monitoração hemodinâmica invasiva, ventilação mecânica básica e terapia nutricional. O principal objetivo é demonstrar, através da prática simulada em manequins, a rotina de procedimentos desta especialidade, permitindo ao aluno a execução e familiarização com diversas situações e procedimentos de uma UTI ${ }^{15}$. As principais vantagens do treinamento com o recurso da simulação são que os estudantes podem exercitar práticas e procedimentos comuns ou incomuns, quantas vezes forem necessárias, permitindo-se o erro, evitando-se o risco ao paciente e não expondo o aluno ao estresse de praticar em situação real, embora a simulação busque o máximo de realismo possível. Tais iniciativas são de fundamental importância na formação dos futuros médicos brasileiros. Contudo, é necessário que as escolas médicas não apenas estimulem tais atividades, mas que também assumam a responsabilidade da formação generalista de nossos profissionais.

\section{CONCLUSÃO}

O presente estudo revelou um alto interesse em Ml entre os estudantes de Medicina de duas escolas baianas. Contudo, mais da metade dos estudantes nunca realizou estágio em UTI. Este fato pode ter contribuído para o aumento observado na segurança do médico em formação, frente a um paciente crítico, uma vez que tal confiança esteve associada à realização de estágio em UTI, muitos dos quais foram apenas extracurriculares. Além de revelarem o interesse dos estudantes na especialidade, os resultados apresentados apontam para possíveis falhas na abordagem na Medicina Intensiva em duas tradicionais escolas médicas brasileiras, o que pode servir para subsidiar estratégias de melhoria do processo de ensino-aprendizagem da MI nestas faculdades.

\section{REFERÊNCIAS}

01. Goldwasser R - Educação em medicina intensiva: uma tarefa para a AMIB e as universidades? Atualidades AMIB, 2003;27:1.

02. Harrison GA, Hillman KM, Fulde GW et al - The need for undergraduate education in critical care. (Results of a questionnaire to year 6 medical undergraduates, University of New South Wales and recommendations on a curriculum in critical care). Anaesth Intensive Care, 1999;27: 53-58.

03. Clarke GM, Harrison GA - The Training/Examination Programme in In- 
tensive Care, Australian and New Zealand College of Anaesthetists. 1. Training. Anaesth Intensive Care, 1993;21:848-853.

04. Moraes APP - Terapia intensiva na graduação médica: nova abordagem, antigas reflexões. Rev Bras Educ Med, 2004;28:81-81.

05. Ayres SM, Messmer J, Wasserman A. Undergraduate education in critical care medicine. Crit Care Med, 1992;20:1506-1507.

06. Buchman TG, Dellinger RP, Raphaely RC et al - Undergraduate education in critical care medicine. Crit Care Med, 1992;20:1595-1603.

07. Taquette SR, Costa-Macedo LM, Alvarenga FBF - Currículo paralelo: uma realidade na formação dos estudantes de medicina da UERJ. Rev Bras Educ Med, 2003;27:171-176.

08. Yamano EYS, Carvalho RKN, Ferreira MS et al - Estágios extracurriculares do estudante de medicina da UFPA, em: Congresso Brasileiro de Educação Médica, 2001, Belém. Anais do XXXIX Congresso Brasileiro de Educação Médica, Associação Brasileira de Educação Médica, 2001;298.

09. Brasil. Mistério da Educação. Conselho Nacional de Educação. Minuta de Resolução, de 3 de outubro de 2001. Institui as Diretrizes Curriculares Nacionais dos Cursos de graduação em Medicina. Boletim ABEM 2001;9-11.
10. Shen J, Joynt GM, Critchley LA et al - Survey of current status of intensive care teaching in English-speaking medical schools. Crit Care Med, 2003;31:293-298.

11. Rogers $\mathrm{PL}$, Jacob H, Rashwan AS et al - Quantifying learning in medical students during a critical care medicine elective: a comparison of three evaluation instruments. Crit Care Med, 2001;29:1268-1273.

12. Goldwasser R - Como está a medicina intensiva na graduação médica? Atualidades AMIB 2003;27:2.

13. Frankel HL, Rogers PL, Gandhi RR et al - What is taught, what is tested: findings and competency-based recommendations of the Undergraduate Medical Education Committee of the Society of Critical Care Medicine. Crit Care Med, 2004;32:1949-1956.

14. Angus DC, Kelley MA, Schmitz RJ et al - Caring for the critically ill patient. Current and projected workforce requirements for care of the critically ill and patients with pulmonary disease: can we meet the requirements of an aging population? JAMA 2000;284:2762-2770.

15. AMIB - Curso de Introdução à Medicina Intensiva. http://www.amib.com. $\mathrm{br} /$ paginasdinamicas/controller?command=MontarPagina\&id_pag=92 (acessado em 08/2007) 